\title{
Percurso desenvolvimental dos portadores da perturbação de HIPERATIVIDADE COM DÉFICIT DE ATENÇÃO
}

\author{
COURSE OF DEVELOPMENT OF ATTENTION DEFICIT HYPERACTIVITY DISORDER
}

Joaquim RAMALHO ${ }^{1}$

RESUM O : a Perturbação de Hiperatividade com Déficit de A tenção (PHDA) constitui uma perturbação com características desenvolvimentais de caráter crônico, que embora seja atenuada com o decorrer da idade, nomeadamente na sua componente hipercinética e impulsiva, a continuidade da presença de sintomatologia de desatenção afeta as capacidades de resposta ao meio ao longo de todo o ciclo vital. Estudos efetuados recentemente têm vindo a demonstrar que entre 50 e $80 \%$ dos sujeitos que manifestaram PHDA durante a infância, experimentam ainda dificuldades significativas relacionadas com esta perturbação durante a fase adulta. Desta forma, o presenteartigo tem como objetivo apresentar o percurso desenvolvimental dos portadores desta perturbação, desdea primeira esegunda infância atéà vida adulta, demonstrando como esta perturbação tem implicações cognitivas, emocionais e sociais e relevantes ao longo de todo o ciclo vital.

PALAVRAS-CHAVE: Educação Especial; perturbação de hiperatividade com déficit de atenção (PHDA); impulsividade; hiperatividade.

ABSTRACT: TheAttention Deficit Hyperactivity Disorder (ADHD) is a disorder with a chronic developmental nature. Although it attenuates with age, particularly its hyperkinetic and impulsive aspects, the continuous presence of attention deficit symptoms affects the capacity of responding to environmental stimuli throughout life. Recent studies have shown that between 50 and 80 per cent of subjects who revealed ADHD in their childhood, have experienced significant difficulties connected with this disorder in their adulthood. Thus, the present articleaims to present the devel opmental course of subjects with ADHD, from infancy to adulthood, to demonstrate that this disorder significantly affects not only the first years but the wholelife cycle.

KEYWO RD S: Special Education; Attention Deficit Hyperactivity Disorder (ADHD); Impulsivity; hyperactivity.

\section{INTRODUÇÃO}

A Perturbação deHiperatividade com Déficit deA tenção (PHDA), pela sua natureza crônica, correspondea uma séria problemática, devido às implicações quelhe estão associadas, nomeadamentea nível cognitivo, familiar esocial, já que os comportamentos destes são caracterizados por fortes componentes de impulsividade, de desatenção e de atividade motora excessiva, que acabam por ter uma enorme repercussão em termos acadêmicos e de relacionamento interpessoal.

Estes aspectos têm legado na PHDA uma importância nunca antes sentida pela família, pela escola e pela sociedade, mas também por parte dos

\footnotetext{
${ }^{1}$ Faculdade de Ciências Humanas e Sociais, Universidade Fernando Pessoa. Porto - Portugal. ramalho@ufp.edu.pt
} 
investigadores, passando a ser considerada como um problema com um focus de análise multidisciplinar.

Uma das primeiras referências às perturbações da atenção data definais do séc. XIX, quando o investigador Ribot, em 1889 (BA RKLEY, 1982), mencionou que são classificados de distraídos aqueles sujeitos cuja inteligência é incapaz de secentrar deuma forma estável passando incessantemente de uma ideia para outra. Refereo autor quetambém seaplica a casos em que os sujeitos manifestam escassa atenção em rel ação a acontecimentos externos.

Uma outra rel evante referência surge em 1902, quando o investigador Still descreveu um grupo de 20 crianças com diversos graus de agressividade, de hostilidade, deconduta desafiante, de desatenção edeH iperatividade(HERREROS et al. 2002), com o objetivo de conceptual izar e val idar uma síndrome que possa agrupar este conjunto de sintomas (PEÑ A; MONTIEL-NAVA, 2003).

As aproximações científicas ao tema da PHDA, duranteos anos 30 e 40 do século XX, derivadas das investigações de caráter essencialmente bioquímico, começaram por designar esta por Disfunção Cerebral Mínima, que designa uma perturbação no desenvolvimento do cérebro infantil, na qual as associações neuronais, responsáveis por determinadas funções, não maturam deacordo coma idade da criança, estabelecendo conexões entre si, apenas de uma forma descontinuada einsatisfatória (VECIANA, 2002). Estes estudos partiam do prinćípio de que existe uma disfunção cerebral que está na gênese de determinados efeitos podem originar perturbações psíquicas, mais especificamente atividade motora excessiva, ansiedade, impulsividade ou falta deatenção.

Posteriormente, as aproximações à PHDA passaram particularmentea incidir sobre a tipologia comportamental de atividade motora excessiva, a qual era vista como o traço característico do sujeito, que ocorria em qualquer circunstância e com uma significatividade acentuada (BARKLEY, 1982; BLÁZQUEZ-ALMERÍA et al., 2005; PEÑ A; MONTIEL-NAVA, 2003).

Nos finais dos anos 70 e inícios dos anos 80 surgem os manuais de diagnóstico e de classificação, como sejam o DSM-II (1968), a CIE-9 (1978) com o intuito de tornar a denominação consensual, como reação hipercinética da infância, para o DSM-II (1968) e como síndrome hipercinético da infância para a CIE-9 (1978), começando também a ser desenvolvidos os primeiros modelos explicativos da referida perturbação (HERREROS et al., 2002).

No decorrer da década de 80, surge, através do autor Barkley (1982), uma nova visão da PHDA. Trata-se de uma alternativa ao modelo atencional , que na sua primeira época, se baseia fundamental mente no fenômeno da desinibição comportamental, apoiado nos trabalhos realizados por Skinner. Como consequência deste fato, no início dos anos 90, começam a ser valorizados não só os aspectos biológicos e comportamentais, mas também os aspectos mais cognitivos, nomeadamente o déficit de atenção. 
A tualmente, a definição e a configuração da PHDA estão envoltas em al guma discussão. As diferentes classificações de diagnóstico como sejam a CIE10 (1992), da Organização Mundial deSaúde, ou o DSM-IV (1996) e o DSM-IV-TR (2002), da Associação A mericana de Psiquiatria, diferem na sintomatologia e diferem também no val or que os sintomas apresentam na al tura de estabelecer um diagnóstico. O DSM-IV (1996) eo DSM-IV-TR (2002) consideram queos principais sintomas são o déficit de atenção, a H iperatividade e a impul sividade, enquanto que a $\mathrm{CIE}-10$ ratifica unicamente os dois primeiros sintomas. Esta conjuntura contribui, naturalmente, para que exista certa ambiguidade edesordem no modo dereal izar um diagnóstico apropriado.

Segundo Associação A mericana de Psiquiatria (2002) a PHDA é uma perturbação que ocorre habitual mente na primeira e na segunda infância ou na adolescência e está inserida no quadro de perturbações disruptivas do comportamento e de déficit de atenção.

A PHDA compreendetrês subtipos distintos, quesão: (1) PHDA, Tipo Misto (PHDA-M) ondea agitação psicomotora eo déficit deatenção estão presentes eambos são significativos (CARDO; SERVERA-BA RCELÓ, 2005; SELL-SALAZAR, 2003; VA N-WIELIN K, 2005); (2) PHDA, Tipo H iperativo-Impulsivo (PHDA-HI), onde são relevantes a H iperatividadee impulsividade; (3) PHDA, Tipo Desatento (PHDA-D), no qual predomina a desatenção, em que estas crianças são referidas essencialmente como distraídas (VAN-WIELINK, 2005) e onde a Hiperatividade não é significativa (DOYLE et al . 2001; SELL-SA LAZA R, 2003). A estes subtipos, estão associadas, entre outras, determinadas problemáticas de cariz comportamental (desatenção, déficit de autocontrole e inquietação), social (dificuldades de relacionamento interpessoal), cognitivo (dificuldades de seleção emanutenção da codificação de estímulos), acadêmica (baixo rendimento escolar, problemas de comportamento), emocional (baixa autoestima, baixa tolerância à frustração, alterações de humor) e físicas (alergias, alterações do sono, baixa coordenação motora). (MARTINEZ, 2004).

Embora o diagnóstico da PHDA resulte, por vezes, em características difíceis de reconhecer, existe consenso científico quando sefal a da sua prevalência, situando-a, na população escolar, entre os 3\% eos 5\% (A RTIGAS-PALLARÉS, 2003; CAPILLA-GONZÁLEZ et al., 2005; CARDO; SERVERA-BARCELÓ, 2005; MAYNARD; TYLER; ARN OLD, 1999; VECIANA, 2002).

Segundo Van-Wielink (2005), os estudos realizados em diversas comunidades indicam queexistem cerca de 17 casos com PHDA em cada 100 crianças. Os al unos queestão em idade escolar apresentam uma frequência de 5 a 9 por cada 100 crianças (HERREROS et al., 2002; MAYNARD; TYLER; ARN OLD, 1999; VANWIELINK, 2005). Para os autores Cardo eSevera-Barceló (2005) utilizando os critérios dediagnóstico do DSM-IV , a maioria dos autores estima uma prevalência global da PHDA, nas suas diversas formas, de 3 a $7 \%$, existindo, no entanto, uma margem de variabilidadeem função da idade, do sexo, do meio sociocultural ou dos subtipos. 
A respeito das diferenças entre gênero, embora exista alguma discrepância (CORNEJO et al., 2005), existe coincidência quanto ao fato de ela apresentar maior prevalência nos sujeitos do sexo masculino do que nos do sexo feminino (CARDO; SERVERA-BA RCELÓ, 2005). Segundo Van-Wielink (2005), existe uma percentagem de 5:1, estando dependenteda população queéestudada, podendo essa percentagem ir de 10:1, em investigações realizadas em clínicas privadas, ou em 3:1 em estudos realizados abertos e baseados na comunidadeem geral .

Segundo Brown (2003) e García-Ogueta (2001), os fatores queexplicam esta diferença entre os sexos devem-se a fatores como a aplicação desigual dos critérios diagnósticos habitual mente utilizados, as razões educativas e cul turais e também as diferenças ao nível das características físicas, mas de igual forma se descobriu recentemente (VAN-WIELINK, 2005) que a PHDA existe em todas as populações estudadas, sendo estas muito diferenciadas ao nível da área geográfica ou estrato econômico-social.

Em relação à idade, a maioria dos estudos revela que a prevalência é maior em idades inferiores estimando-se que em $75 \%$ dos casos se verifica antes dos 5 anos (VAQUERIZO-MADRID, 2005) ou entre os 6 e os 9 anos (CARDO; SERVERA-BARCELÓ, 2005). Segundo Kooij (2009) cerca de 2 em cada 10 adultos manifesta PHDA.

\section{Percurso desenvolvimental dos portadores da PHDA}

A pesar de al guns dos estudos longitudinais real izados terem vindo a procurar demonstrar que embora a sintomatologia da PHDA venha, tendencialmente, a diminuir com o avançar da idade, estetipo de perturbação tem vindo a receber um caráter particularmente crônico (MIRANDA-CASAS etal., 2000). Segundo Kooij (2009), a PHDA é uma perturbação de origem seguramente pré natal, que se manifesta cedo, tipicamente no início da idade escolar e cujas consequências se prolongam, quase sempre, por toda a vida.

Estudos recentemente efetuados têm vindo a demonstrar que entre 50 e $80 \%$ dos sujeitos que manifestaram PHDA durante a infância, experimentam ainda dificuldades significativas relacionadas com esta perturbação durantea fase adulta (CARDO; SERVERA-BARCELÓ, 2005; VAQUERIZO-MADRID, 2005). Desta forma, procura-se, deseguida, apresentar o percurso desenvolvimental dos sujeitos com PHDA desde a primeira infância atéà vida adulta, demonstrando como esta perturbação tem implicações cognitivas, emocionais esociais erelevantes ao longo detodo o ciclo vital.

\section{Primeira infância}

Segundo Vaquerizo-Madrid (2005), em cerca de $75 \%$ dos casos, a PHDA éproduzida e demonstrada antes dos 3 ou 4 anos de idade. A informação oferecida 
por histórias clínicas, indica que uma significativa percentagem destes sujeitos fora bebês com enormes dificuldades em realizar novas adaptações, criando contínuas resistências a tudo que al terava a rotina diária.

Segundo Miranda-Casas, Luz e Fernández (2001), os pais recordam estes bebês como reagindo de um modo despropositado perante os estímulos do meio, como sendo irritáveis, com uma necessi dade de atenção contínua, com uma excessiva atividade psi comotora e com uma baixa tolerância à frustração.

Outros autores consideram queestes bebês manifestam dificuldades nos padrões do sono (BETA NCOURT-JIMÉNEZ; JIMÉNEZ-LEÓN; JIMÉNEZBETANCOURT, 2006) e na alimentação (GARCÍA-OGUETA, 2001). Estes bebês dormem, comprovadamente, menos do que os bebês com a mesma idade, tendo noites bastante instáveis, com choro e gritos frequentes, manifestando também padrões irregulares de respi ração e de batimentos cardíacos na fase REM. No que respeita à al imentação, são relatados bastantes casos de bebês com dificul dades de transição e adaptação aos alimentos sólidos depois do abandono do biberão, particularmenteem bebês que em fase posterior foram diagnosticados com PHDA (CHEON etal., 2003; MIRANDA-CASASetal., 2006; VAQUERIZO-MADRID, 2005).

Este padrão tipificado de comportamentos de sujeitos com PHDA durante a sua primeira infância origina, inclusive nos pais mais competentes e responsáveis, sentimentos deimpotência pessoal, frustração, o queacaba por afetar a sua relação com os filhos, conduzindo à aplicação de normas disciplinares, nuns casos, bastante permissivas e bastantes punitivas noutros (ROGERS, et al. 2009).

Segunda infância

Na segunda infância é difícil estabelecer a distinção entre crianças com esem sintomatologia PHDA, já que existem diversos padrões comuns entre ambos. No entanto, naqueles em cujo diagnóstico revela a presença desta perturbação patenteiam-se comportamentos significativos de ansiedade, déficit de atenção e deexcessiva atividade motora.

Uma das princi pais indicações da presença da PHDA está relacionada com as capacidades de focal ização e manutenção da atenção perante as tarefas. Estes sujeitos, especialmente quando estão perantetarefas mais monótonas ecom períodos mais alongados de tempo (FERNÁ NDEZ, 2003), procuram desistir da realização da tarefa ou alterná-la com a realização de outras tarefas.

Uma outra sintomatologia diz respeito ao comportamento e posição perante atividades lúdicas. As crianças com PHDA manifestam uma maior curiosidade perantejogos novos, no entanto aborrecem-se com enormefacilidade, mudando de jogo de forma constante. Quando jogam com as outras crianças, procuram al terar as regras, têm dificul dades em lidar com a derrota, apresentam dificuldades em atividades de grupo que impliquem cooperação e procuram discutir com os companheiros deatividade, acusando-os, por vezes, da sua própria 
derrota (BROWN , 2003; GARCÍA-OGUETA, 2001; GRATCH, 2000; MIRANDACASAS; LUZ; FERNÁNDEZ, 2001; MIRANDA-CASAS et al., 2006). São ainda denotados comportamentos de base de conduta desobediente, desafiador e oposicional (RAMALHO, 2009).

Em nível afetivo e emocional, as crianças com PHDA manifestam dificuldades em estabel ecer relações afetivas com outros sujeitos, tendo também dificuldades em reconhecer posturas, queéresultante de déficit dereconhecimento de aspectos relevantes da comunicação não-verbal (MIRANDA-CASAS; LUZ; FERN ÁN DEZ, 2001) eimaturidadedalinguagem expressiva (FERNÁN DEZ, 2003; GARCÍA-OGUETA, 2001).

Etapa escolar

Quando a criança com PHDA entra formalmente na fase escolar começam a ser mais patenteadas as dificuldades posturais, como sejam, o ter que permanecer sentado durante longos períodos de tempo.

Nesta fase, são evidenciadas dificuldades de caráter cognitivo, que resultam de fatores diversos como a impulsividade e deficientes capacidades de focalização e manutenção da atenção (RAM L LHO, 2009). Estes aspectos acabam por acarretar situações negativas em termos de funcionamento acadêmico e comportamental (CHAPMAN; TUN MEER; PROCHNOW, 2000), já que estes sujeitos acabam por dedicar menos tempo às atividades escolares, utilizam menor esforço para conseguirem alcançar os seus objetivos e utilizam estratégias de aprendizagem com níveis de el aboração e execução mais simples (BROWN , 2003).

Segundo Gratch (2000), os sujeitos com PHDA manifestam padrões executivos bastante variáveis ao longo do tempo, de forma que por vezes são até capazes de realizar as tarefas de uma forma correta, terminá-las no tempo previamente estabelecido e com uma postura adequada e, noutras vezes não só não conseguem terminar, como, por vezes, nem iniciam as tarefas. Estas crianças perdem também, muitas vezes, derivado deal guma desorganização, o seu material escolar, como sejam, por exemplo, os livros.

São latentes também as dificuldades de caráter socioemocional. Estes sujeitos apresentam fracos pad rões derelacionamento com os pares ecom as figuras significativas (RAMALHO, 2009), dificuldades de partilha, de cooperação e interação com os seus col egas (GA RCÍA-OGUETA, 2001).

Segundo García-Ogueta (2001), como consequência das dificuldades de rendimento escolar e de aceitação social, os sujeitos com PHDA vão passar a manifestar sentimentos de frustração e incompetência, dificuldades de aceitação pessoal evão desenvolver níveis deautoconceito bastante baixos.

É decorrente de todos estes fatos que os professores começaram a procurar razões para o fato de estas crianças apresentarem um baixo rendimento escolar (BEN ASSINI, 2005; MIRANDA-CASAS; LUZ; FERNÁNDEZ, 2001). Daí 
que seja, nesta fase, que mais se procura a realização de um diagnóstico que comprove a presença da PHDA.

\section{A dolescência}

A fase da adolescência é marcada por fortes mudanças a nível físico, perceptivo eemocional etodas estas mudanças podem provocar al terações internas, nomeadamente ao nível da autoestima e o autoconceito, mas também alterações externas, como sejam, o relacionamento familiar ecom os pares (ROGERSet al ., 2009).

Estas alterações provocam, não raras vezes, manifestações comportamentais decariz desafiador eoposicional, particularmenteface às figuras de autoridade (GARCÍA-OGUETA, 2001). N os sujeitos com PHDA, todas estas mudanças aumentam bruscamente, já que para além demanifestarem as al terações associadas à idade, manifestam também as al terações associadas à perturbação e os comportamentos desafiadores e oposicionais e os transtornos da conduta são ainda mais notórios (BEN ASSINI, 2005).

Segundo Miranda-Casas et al. (2006), os problemas que os sujeitos com PHDA manifestavam em nível social quando eram mais jovens, são agora ainda mais preocupantes, já que para além de apresentarem problemas de conduta bem mais severos, trazem da infância a presença de um estatuto social de rejeição.

Os sujeitos com PHDA manifestam também problemas ao nível do rendimento escolar, uma vez que são reveladas mais dificuldades ao nível da execução, da destreza manual, da aritmética e da compreensão da leitura. A presentam também um número significativamente maior de expulsões e suspensões escolares, de reprovações e de abandono escolar (BROWN, 2003; MIRANDA-CASAS; LUZ; FERNÁNDEZ, 2001).

Desta forma e, decorrente do fato da formação do autoconceito depender, nesta fase, quase exclusivamente dos sucessos escolares, sociais, artísticos e/ ou desportivos, domínios em queos sujeitos com PHDA manifestam claras dificuldades deafirmação, uma percentagem el evada destes adolescentes não têm autoconfiança, crescendo com sentimentos deautodesval orização, deindefesa ecom sintomatologia depressiva (MIRANDA-CASAS; LUZ; FERNÁNDEZ, 2001; VAN-WIELINK, 2005).

Vida adulta

A falta de reconhecimento e o subdiagnóstico de PHDA nos adultos é tanto mais preocupante quanto se trata de uma perturbação tratável e, mais ainda, quando o tratamento adequado se pode traduzir numa melhoria extraordinária da qual idade de vida dequem del a padecee dos quelhe são próximos (KOOIJ, 2009).

$\mathrm{Na}$ generalidade, os comportamentos com características predominantementehiperativas eimpulsivas vão sendo atenuados atéà faseda vida adulta (BROWN , 2003), no entanto continuam a persistir padrões comportamentais 
de déficit deatenção, associados a comportamentos ansiedade einquietude, de falta de premeditação, de descuido e falta de verificação, de desorganização, de falta de planificação, debaixamemória a curto prazo ededificuldadesem estabelecer emanter uma rotina diária adequada, particularmenteao nível laboral.

Diversos autores têm também vindo a ser encontrados padrões de relação entrea PHDA eo déficit de realização cognitiva (ROSE et al., 2009), porque não utilizam o seu potencial da melhor forma.

Os dados clínicos revel am que os adultos quecontinuam a manifestar alguma da sintomatologia da PHDA, têm menos escolaridade, uma procura constante de elevada estimulação externa têm uma pior capacidade de gestão monetária, organizam com lacunas as suas tarefas domésticas, têm dificuldades em real izar atividades com os filhos, são menos autônomos, menos organizados, pelo que têm dificuldades em realizar tarefas sem uma supervisão eficiente e manifestam também uma progressão no estatuto educativo e ocupacional significativamentemais lenta (MIRANDA-CASAS; LUZ; FERNÁNDEZ, 2001).

Segundo Rose et al. (2009), os sujeitos com PHDA, na fase adulta, manifestam uma procura constante de elevada estimulação externa, mudanças frequentes de humor sem razão aparentee baixa autoestima.

\section{Conclusão}

Este artigo procurou fornecer um melhor conhecimento do percurso dos sujeitos com PHDA ao longo da vida, de forma a contribuir para atenuar al gumas das comorbilidades resultantes desta perturbação.

Com os dados revelados, éimportante quese perceba queéimportante, cada vez mais, tratar eficazmente e mel horar a qualidade de vida destes sujeitos ao longo do ciclo vital. É essencial o desenvolvimento de instrumentos de diagnóstico e de intervenção na PHDA para adultos.

A PHDA, durante anos a mais, foi encarada como uma perturbação apenas da infância e adolescência, sem que fossem devidamente estudadas e aprofundadas as suas implicações futuras, nomeadamentena faseadulta. Os dados que não enal tecem os seus comprometimentos durante todo o ciclo vital estão absoluta e comprovadamenteerrados, já que, como foi explicitado por diferentes autores e perspectivas científicas, esta perturbação acarreta problemáticas significativas e desajustantes ao longo de toda a vida.

Para finalizar esegund o o autor Josel evich (2000), ficam al guns dados rel evantes, que comprovam a pertinência de estudos neste âmbito: cerca de 30 a $50 \%$ dos sujeitos com PHDA têm grandes probabilidades de repetir o ano de escolaridade, nem que seja por uma só vez; mais de $20 \%$ têm forte probabilidade de serem suspensos ou mesmo expulsos da escola; 50\% destes apresentam dificuldades de relacionamento com os colegas de escola e/ ou de trabal ho; $35 \%$ 
não completam os estudos secundários; $70 \%$ apresentam muitas dificuldades no controlo dos impulsos e na resistência à frustração durante todo o ciclo vital; e cerca de $60 \%$ continuam a apresentar, durante a vida adulta, problemas psicossociais resultantes da existência da perturbação durantea infância.

\section{RefERÊnCIAS}

APA. D SM -IV : manual de diagnóstico e estatística das perturbações mentais. Lisboa: Climepsi Editores. 1996.

. D SM -IV -TR : manual de diagnóstico e estatística das perturbações mentais. 4. ed. Texto revisto. Lisboa: Climepsi Editores. 2002.

ARTIGAS-PALLARÉS, J. Comorbilidad en el trastorno por déficit de atención y hiperactividad. N eurología, v.36 (supl. 1), S68-S78, 2003.

BA RKLEY, R. Guidelines for defining hyperactivity in children. Attention Deficit Disorder with Hyperactivity. In: LAHEY B. B.; KAZDIN (Eds.). A dvances in Clínical Child Psychology. New York: Plenum Press. 1982.

BENASSINI, O. Trastornos de la atención: origen, diagnóstico, tratamiento y enfoque psicoeducativo. Sevilla: Editorial Eduforma. 2005.

BETANCOURT-JIMENEZ, Y., JIMENEZ-LEON, J. ; JIMENEZ-BETAN COURT, C. Trastorno por déficit de atención e hi peractividad y trastornos del sueño. N eurología, v.42, n.2, S37S51, 2006.

BLÁZQUEZ-ALMERÍA, G. et al. Resultados del cribado dela sintomatología del trastorno por déficit de atención con o sin hiperactividad en el ámbito escolar mediante la escala EDAH. N eurología, v.41, n.10, p.586-590, 2005.

BROWN, T. Trastornos por déficit de atención y comorbilidades en niños adolescentes y adultos. Barcelona: Editora Masson. 2003.

CAPILLA-GONZÁLEZ, A. et al. Nuevas aportaciones a la neurobiología del trastorno por déficit de atención con hiperactividad desde la magnetoencefalografia. N eurología, v.40 (Supl 1), S43-S47, 2005.

CARDO, E.; SERVERA-BARCELÓ, M. Prevalencia del trastorno de déficit de atención e hiperactividad. N eurología. v.40 (supl1), S11-S15. 2005.

CORNEJO, J. et al. Prevalencia del trastorno por déficit deatención-hiperactividad en niños y adolescentes colombianos. N eu rología, v.40, n.12, p.716-722, 2005.

CHAPMAN, J.; TUNMEER, W.; PROCHNOW, J. Early reading-related skills and performance, reading self concept and the development of academic self-concept: a longitudinal study. Journal of Educational Psychology, v.92, n.4, p.703-708, 2000.

$\mathrm{CHEON}$, K. et al. Dopamine transporter density in the basal ganglia assessed with IPTSPET in children with attention Deficit Hyperactivity Disorder. Eur J N ucl M ed M ol Imaging, v.30, p.306-311, 2003.

DOYLE, A . et al. Separating attention deficit hyperactivity disorder and learning disabilities in girls: a familial risk analysis. A m J Psychiatry, v.158, p.1666-1672, 2001.

FERNÁNDEZ, A.P roblemas delenguajecon estudiantes con déficit atencional. Valencia: Servei dePublicacions. 2003. 
GARCÍA-OGUETA, M. Mecanismos atencionales y síndromes neuropsicológicos. N eurología, v.32, n.5, p.463-467, 2001.

GRATCH, L. El trastorno por déficit de atención: clínica, diagnóstico y tratamiento en la infancia, la adol escencia y la adultez. Buenos Aires: Editorial Medica Panamericana. 2000. HERREROS, O. etal. Evaluación deniñosy adolescentes con trastorno por déficit deatención con hiperactividad/ Trastornos hipercinéticos. Psiquiatría Infanto-Juvenil, v.19, n.4, p.199214, 2002.

JOSELEVICH, E. Síndromede déficit deatención con o sin hiperactvidad. Buenos Aires: Editorial Paidós. 2000.

KOOIJ, S. A perturbação de hiperactividade com déficit de atenção em adultos. Lisboa: Medicina XXI. 2009.

MARTINEZ, M. D ificultades ligadas a la hiperactividad y al deficit de atención. Necessidades Educativas Especiales. Madrid: McGraw-Hill. 2004.

MAYNARD, J.; TYLER, J.; ARNOLD, M. CoOcurrence of attention deficit disorder and learning disability: an overview of research. Journal of Instructional Psychology. 1999.

MIRANDA-CASAS, A et al. Dificultades en el aprendizaje de matemáticas en niños con trastorno por déficit de atención e hi peractividad. N eurología, v.42, p.2, S163-S170, 2006.

MIRANDA-CASAS, A . et al. Intervención psicoeducativa en estudiantes con trastorno por déficit de atención con hiperactividad. N eurología Clínica, v.1, p.203-216. 2000.

MIRANDA-CASAS, A.; LUZ, L.; FERNÁNDEZ, S. Trastornos por déficit de atención con hiperactividad: una guía práctica. Málaga: Ediciones Aljibe. 2001.

PEÑ A, J.; M ONTIEL-NAVA, C. Trastorno por déficit deatención/ hiperactividad: ¿mito o realidad? N eurología, v.36, n.2, p.173-179, 2003.

RAMALHO, J. P sicologia e psicopatologia da atenção. Braga: Edições APPACDM , 2009.

ROGERS, $M$. et al. Parental involvement in children's learning: Comparing parents of children with and without Attention-Deficit/ Hyperactivity Disorder (ADHD). Journal of School Psychology, v.47, p.167-185, 2009.

ROSE, E. et al. Neuropsychological characteristics of adults with comorbid ADHD and borderline/ mild intellectual disability. Research in D evelopmental D isabilities, v.30, p.496502, 2009.

SELL-SALAZAR, F. Síndrome de hi peractividad y déficit deatención. N eurología, v.37, n.4, p.353-358, 2003.

VAN-WIELINK, G. D éficit deatención con hiperactividad: estrategias, habilidades, diagnóstico y tratamiento. México: Editorial Trillas. 2005.

VAQUERIZO-MADRID, J.; ESTÉVEZ-DÍAZ, F.; DÍAZ-MAÍLLO, I. Revisión del modelo de alerta e intervención psicolinguística en el trastorno por déficit de atención e hi peractividad. N eurología, v.42, n.2, S53-S61, 2006.

VECIANA, J. Los trastornos de la atención y la hiperactividad: diagnóstico y tratamiento neurofuncional y causal. Barcel ona: Ediciones Lebón, 2002.

Recebido em: 20/ 04/ 2010

Reformulado em: 17/ 07/ 2010

A provado em: 19/ 07/ 2010 